\title{
Study of the protective effect of dexamethasone on cisplatin- induced ototoxicity in rats ${ }^{1}$
}

Isabelle Oliveira Jatai Capelo', Avner Marcos Alves Batista", Yuri Neyson Ferreira Brito", Krissia Braga Diniz", Gerly Anne de Castro Brito"I', Marcos Rabelo de Freitas ${ }^{\text {IV }}$

'MSc, Department of Surgery, Universidade Federal do Ceará (UFC), Fortaleza-CE, Brazil. Acquisition and interpretation of data, technical procedures, manuscript preparation.

"Graduate student, UFC, Fortaleza-CE, Brazil. Acquisition of data, technical procedures.

IIIPhD, Associate Professor, Morphology Department, School of Medicine, UFC, Fortaleza-CE, Brazil. Analysis and interpretation of data, technical procedures, critical revision.

IVPhD, Associate Professor, School of Medicine, UFC, Fortaleza-CE, Brazil. Conception, design, intellectual and scientific content of the study; analysis and interpretation of data; critical revision.

\section{Abstract}

Purpose: To evaluate the ability of dexamethasone to protect against cisplatin (CDDP)induced ototoxicity.

Methods: Male Wistar rats were divided into the following three groups: 1) Control (C): 6 animals received intraperitoneal (IP) saline solution, $8 \mathrm{ml} / \mathrm{kg} /$ day for four days; 2) C + CDDP: 11 animals received $8 \mathrm{ml} / \mathrm{kg} /$ day of IP saline and, $90 \mathrm{~min}$ after saline administration, $8 \mathrm{mg} / \mathrm{kg} /$ day of IP CDDP for four days; and 3) DEXA15 + CDDP: 11 animals received IP dexamethasone $15 \mathrm{mg} / \mathrm{kg} /$ day and, $90 \mathrm{~min}$ after dexamethasone administration, received $8 \mathrm{mg} / \mathrm{kg} /$ day of IP CDDP for four days.

Results: It was found that dexamethasone did not protect against weight loss in CDDPexposed animals. The mortality rate was comparable with that previously reported in the literature. The auditory threshold of animals in the DEXA15 + CDDP group was not significantly altered after exposure to CDDP. The stria vascularis of animals in the DEXA15 + CDDP group was partially preserved after CDDP exposure.

Conclusions: Dexamethasone at the dose of $15 \mathrm{mg} / \mathrm{kg} /$ day partially protected against CDDPinduced ototoxicity, based on functional evaluation by brainstem evoked response audiontry (BERA) and morphological evaluation by optical microscopy. However, dexamethasone did not protect against systemic toxicity.

Key words: Dexamethasone. Cisplatin. Rats. 


\section{Introduction}

Cisplatin (cis-diamminedichloridoplatinum(II); (DDP) is a chemotherapy widely used in the treatment of pediatric and adult cancers $^{1}$. The side effects of this drug include ototoxicity, nephrotoxicity, gastrointestinal effects, medullary suppression and peripheral neuropathy, which reduce patient quality of life and may result in treatment protocol changes ${ }^{2}$. The ototoxicity induced by CDDP is characterized by bilateral, irreversible, dosedependent hearing loss that initially affects high frequencies and may be accompanied by tinnitus. Approximately $60-80 \%$ of CDDPtreated patients have elevated auditory thresholds, and $15 \%$ will have significant hearing loss ${ }^{3}$.

The antitumor effect of CDDP occurs through the production of reactive oxygen species (ROS), which cause apoptosis, and the increased expression of extracellular regulatory kinase (ERK) and nuclear factor kB (NF-KB), which stimulate proinflammatory cytokine production. The cochlear structures most affected by CDDP are the outer hair cells $(\mathrm{OHC})$, the spiral ganglion and the stria vascularis ${ }^{4}$.

Several drugs have been used to reduce CDDP-induced ototoxicity. Glucocorticoids have shown promise as otoprotective drugs, as they are able to prevent the formation of ROS and inhibit ERK and NF-KB activation ${ }^{5}$. Most studies involving corticosteroids use intratympanic administration with satisfactory results ${ }^{6}$. The objective of this study was to evaluate the otoprotective effect of systemic corticosteroids on CDDP-induced ototoxicity.

\section{Methods}

The project was approved by the Animal Research Ethics Committee (CEPA) under protocol number 127/16.

The study used twenty-eight male Wistar rats weighing 200-260 grams that were kept in cages with free access to food and water and natural sleep and wake cycles.

The animals were divided into three groups:

Group $1(n=6)$ (Control; C): rats were treated on four consecutive days with saline solution at $8 \mathrm{ml} / \mathrm{kg} /$ day (total of $32 \mathrm{ml} / \mathrm{kg}$ ). The animals were evaluated by brainstem evoked response audiometry (BERA) one day before (D0) and four days after (D4) treatment initiation.

Group $2(n=11)(C+C D D P)$ : rats were treated on four consecutive days with saline solution at $8 \mathrm{ml} / \mathrm{kg} /$ day (total of $32 \mathrm{ml} / \mathrm{kg}$ ) and with CDDP at $8 \mathrm{mg} / \mathrm{kg}$ (total of $32 \mathrm{mg} / \mathrm{kg}$ ) 90 min after saline administration. The animals were evaluated by BERA on D0 and D4.

Group $3(n=11)(D E X A 15+$ CDDP): rats were treated on four consecutive days with dexamethasone at $15 \mathrm{mg} / \mathrm{kg} /$ day (total of 60 $\mathrm{mg} / \mathrm{kg}$ ) and with CDDP at $8 \mathrm{mg} / \mathrm{kg} /$ day (total of $32 \mathrm{mg} / \mathrm{kg}) 90 \mathrm{~min}$ after dexamethasone administration. The animals were evaluated by BERA on DO and D4.

Wistar rats underwent deep anesthesia with $80 \mathrm{mg} / \mathrm{kg}$ ketamine combined with 10 $\mathrm{mg} / \mathrm{kg}$ xylazine. A previous otoscopy was performed, and animals with external and middle ear alterations were excluded from the study. Those with normal otoscopy received auditory evaluation (BERA) just prior to drug administration (D0). In all groups, the medications were injected intraperitoneally. In groups 2 and 3, the chemotherapy injection was performed 90 minutes after the initial injection of dexamethasone or saline solution. On the subsequent three days, the drugs were re-administered after the rats were reweighed. Twenty-four hours (D4) after the last injection, the rats were anesthetized, and a new otoscopy was performed to exclude any rats that acquired external or middle ear diseases during the drug administration period. All remaining rats were re-evaluated by BERA. 
Immediately after the last auditory evaluation, the rats were euthanized by decapitation with a custom-made guillotine, and the right temporal bone was removed. The cochlea was dissected for histological preparation.

BERA was performed with the ICS CHARTR EP 200 device (Otometrics-Denmark). After anesthesia, platinum subdermal electrodes were positioned in the vertex (positive), right retroauricular region (negative) and right front leg (ground). ER-3A insertion earphones were coupled to a probe and introduced into the external right ear canal. The stimuli used were rarefaction clicks, released at a rate of 15 per second, with a maximum total of 1000 sweeps and an analysis time of $15 \mathrm{msec}$. The bandwidth used was 0 to $2000 \mathrm{~Hz}$. The stimuli were initiated at $80 \mathrm{~dB}$ sound pressure level (SPL) and progressively decreased until the waves disappeared completely. The lowest stimulus intensity in which wave II appeared was considered to be the electrophysiological auditory threshold.

Cochlear dissection and optical microscopy were performed according to the method described by Freitas ${ }^{23}$. After preparation of the slides, the presence/absence of lesions in the stria vascularis and in the organ of Corti was evaluated and visualized with 400 $x$ magnification in the Leica DM LS 2 optical microscope. Photomicrographs were acquired with Leica 320 digital capture equipment.

The program GraphPad Prism 7.02 was used for statistical analysis. The normal distribution of the sample was evaluated by the Kolmogorov-Smirnov test. The results are expressed as the mean \pm standard error of the mean (MED \pm EPM) for continuous data. The minimum significance accepted was at the $5 \%$ level. Two-way repeated measures ANOVA with the Sidak multiple comparisons test was used to compare animal weight variation, BERA electrophysiological thresholds, and BERA I-V interval values among groups. Fisher's exact test was used to compare stria vascularis and outer hair cells $(\mathrm{OHC})$ morphological changes among groups.

\section{Results}

Systemic toxicity of CDDP

A statistically significant difference was observed in the weights of the animals of all groups between D0 and D4, with $p<0.0001$. The weight decreased in all groups, except in Group C, where there was weight gain (Figure 1).

WEIGHT VARIATION

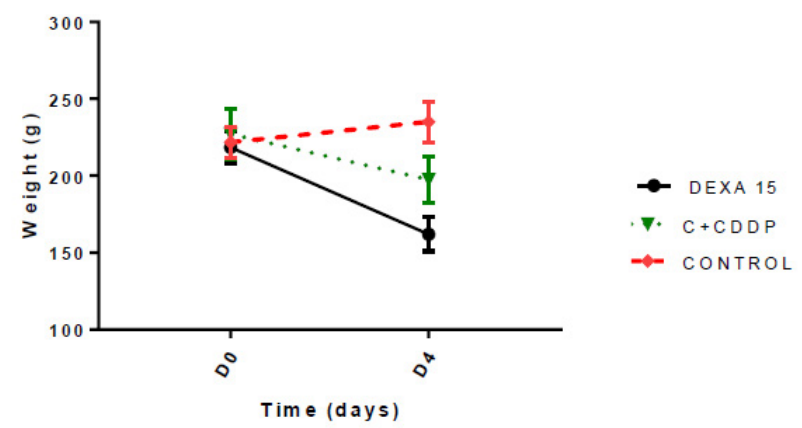

Figure 1 - Graph showing the weight variation for groups 1,2 and 3 , on days 0 (D0) and 5 (D4). $C=$ control. $C+C D D P=$ control + cisplatin ; DEXA15+CDDP = dexamethasone $15 \mathrm{mg}+$ cisplatin.

\section{Functional auditory evaluation}

The BERA test revealed a statistically significant difference between the beginning (DO) and the end of the experiment (D4) in the $C+$ CDDP group ( $p=0.0014$ ) (Figure 2 ). There was no statistically significant difference in the I-V interval between the first (D0) and fifth (D4) days of the experiment in any of the groups (Figure 3). 
BERA THRESHOLD

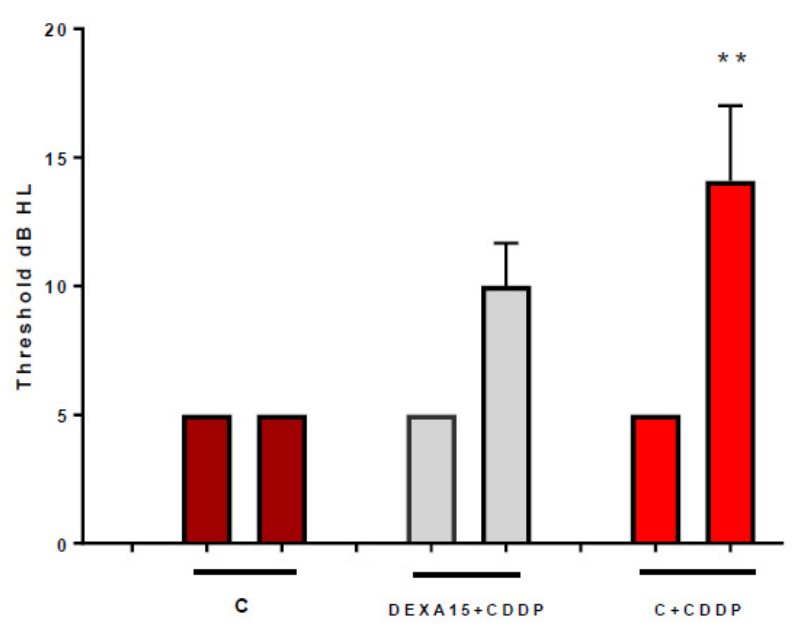

Figure 2-Graph showing the mean eletrophysiologic thresholds of groups 1, 2 and 3 in D0 and D4. The asterisk represents statistical signficance. ANOVASIDAK: ${ }^{*} p=0.0014$. $C=$ control; $C+C D D P=$ control + cisplatin; DEXA15+CDDP = dexamethasone 15mg + cisplatin; BERA = brainstem evoked response audiometry.

\section{BERA}

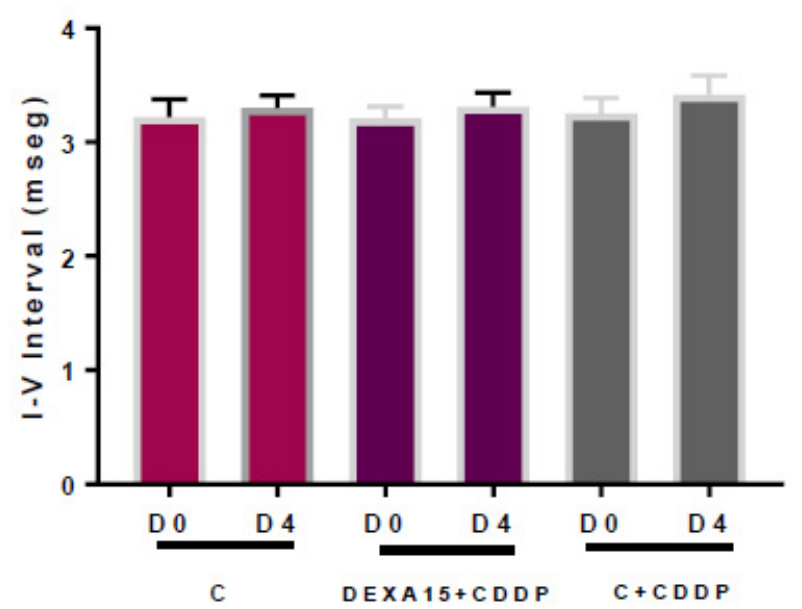

Figure 3 - Graph of the I-V interval expressed as mean \pm standard error of the mean (MED \pm EPM) in days 1 and 5 . There was no statistically significant difference in the I-V interval between the first (D0) and fifth (D4) days of the experiment in any of the groups. C: control; C+CDDP: control + cisplatin; DEXA15 +CDDP: dexamethasone $15 \mathrm{mg}+$ cisplatin. BERA $=$ brainstem evoked response audiometry.

\section{Evaluation by optical microscopy}

In this study, the cochlea of two animals (one from group $\mathrm{C}+\mathrm{CDDP}$ and one from group DEXA15 + CDDP) were lost due to inclusion errors. Therefore, the analysis was conducted with 24 slides including six from group $C$, ten from group $C+C D D P$, and eight from group DEXA15 + CDDP.

\section{Optical microscopy of the stria vascularis}

The stria vascularis was evaluated for presence or absence of retraction in the middle layer. A statistically significant difference was observed only in the $\mathrm{C} \times \mathrm{C}+\mathrm{CDDP}$ group regarding the presence of morphological changes ( $p$ value $<0.05$ ), and this information was obtained through the microscopic analysis of the stria vascularis (Figure 4).

$$
\text { STRIA VASCULARIS HISTOLOGICAL LESION }
$$

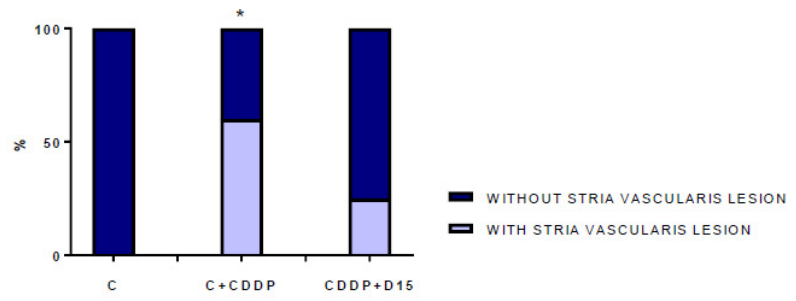

Figure 4 - Graph showing the presence or absence of lesion in stria vascularis, in percentage. The asterisk represents statiscal significance. FISHER 2x2: * $p=0.0338$. C: control; C+CDDP: control + cisplatin; DEXA15 +CDDP: dexamethasone $15 \mathrm{mg}+$ cisplatin.

\section{Optical microscopy of $\mathrm{OHC}$}

Analysis of the optical microscopy data revealed a statistically significant difference between the groups $\mathrm{C} \times \mathrm{C}+\mathrm{CDDP}$ and $\mathrm{C} \times$ DEXA15 + CDDP for the OHC (Figure 5). 
OUTER HAIR CELLS HISTOLOGICAL LESION

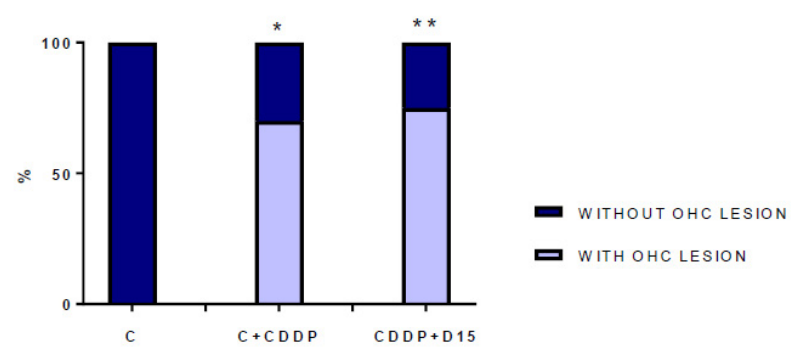

Figure 5 - Graph showing the presence or absence of lesion in $\mathrm{OHC}$, in percentage. The asterisk represents statiscal significance. FISHER $2 \times 2$. * $\mathrm{p}=0.0114 ;{ }^{* *} \mathrm{p}<0.01$. C: control; C+CDDP: control + cisplatin; DEXA15 +CDDP: dexamethasone $15 \mathrm{mg}$ + cisplatin

\section{- Discussion}

CDDP is a chemotherapeutic agent widely used in the treatment of solid tumors, notably head and neck cancers ${ }^{7}$, urogenital system cancers, central nervous system tumors, osteosarcoma, and esophageal cancers ${ }^{8}$. Its use has been limited by a variety of adverse effects such as ototoxicity, nephrotoxicity, neurotoxicity, spinal cord suppression and gastrointestinal effect $^{9}$. Some of the side effects of CDDP can be counteracted by the use of medications and hydration, for example nephrotoxicity and gastrointestinal effect ${ }^{10}$. However, ototoxicity remains one of the side effects that causes significant morbidity and often limits its use ${ }^{11}$.

Glucocorticoids have been evaluated as potential otoprotective drugs based on their anti-inflammatory effects, ionic homeostasis and immunosuppressive effects ${ }^{12}$. Systemic glucocorticoid administration is common and routine in the management of inner ear diseases ${ }^{13}$. Corticosteroids inhibit mitogenactivated protein kinases (MAPKs), important regulators of transcription factors of proinflammatory agents ${ }^{14}$. MAPKs regulate various cellular events, including differentiation, proliferation and apoptosis ${ }^{15}$. ERK, p38 and JNK belong to the family of these mitogen-activated protein kinases and are important components in the signal transduction pathway involved in the production of proinflammatory cytokines and apoptosis ${ }^{16}$. All three MAPKs are also implicated in the NF-KB activation pathway through the phosphorylation of their inhibitor, $\mathrm{IkB}^{17}$. Glucocorticoids may also induce the expression of $\mathrm{lkB}-\alpha$, which suppresses NF$\mathrm{KB}$ by inhibiting the inflammatory cascade ${ }^{18}$. Pharmacological inhibition of ERK is more effective in suppressing the secretion of proinflammatory cytokines and preventing cell death when compared with CDDP inhibition of p38 and $\mathrm{JNK}^{19}$.

Although there are few quantifiable ways of assessing CDDP for systemic toxicity, such as anorexia and diarrhea ${ }^{20}$, objective and measurable parameters such as weight loss and final survival rate have been preferred.

The weight of the animals decreased significantly in all groups, except in the control group where there was weight gain. In the $\mathrm{C}+$ CDDP group, there was a mean weight loss of $13 \%$ in D4, and in the DEXA15 + CDDP group, the weight loss was $25 \%$. Kamimura et al. ${ }^{21}$, using a $16 \mathrm{mg} / \mathrm{kg}$ dose of CDDP, showed a mean weight loss of $24.5 \%$ in Wistar rats, which was similar to that observed in the DEXA15 + CDDP group in this study. Tanaka et al. ${ }^{22}$ found a similar result as described with a $23 \%$ weight loss in animals treated with a dose of $13 \mathrm{mg} /$ kg. However, other authors such as Freitas et al. ${ }^{23}$ found a mean weight loss, after 3 days of evaluation, of $14 \%$ for the dose of $16 \mathrm{mg} / \mathrm{kg}$ of CDDP. In group C, there was an increase in the weight of the animals of approximately $6 \%$, and although the animals were also submitted to the stress of intraperitoneal injection, they did not present diarrhea or reduced appetite like the animals exposed to CDDP.

$$
\text { Intraperitoneal injection of }
$$
dexamethasone was performed 90 min prior 
to administration of CDDP, given that the maximum concentration of glucocorticoid by this route of administration in the perilymph occurs within 2 hours ${ }^{24}$.

The effect of dexamethasone at a dose of $15 \mathrm{mg} / \mathrm{kg}$ on ototoxicity revealed the protective role in the functional evaluation by BERA. There was no statistically significant difference between auditory potentials on D0 and D4 in this group, which demonstrated that corticosteroid use had a protective effect on the action of CDDP with no significant increase in auditory thresholds. In the C + CDDP group, a significant difference was found between auditory potentials on DO and D4, showing the ototoxic potential of CDDP in the C + CDDP group. Sun et al. ${ }^{25}$ also demonstrated that administration of multidose dexamethasone multidoses can significantly protect hearing function. However, most studies using intraperitoneal dexamethasone alone as an otoprotective agent found no significant protection against CDDP ototoxicity through BERA $^{26,27}$.

The $15 \mathrm{mg} / \mathrm{kg}$ dose of dexamethasone plus functional protection, as revealed through the BERA, also revealed histological protection. The present study did not find a statistically significant difference when comparing group $C$ with the DEXA15 + CDDP group in relation to preservation of stria vascularis, demonstrating that there was protection with this dose of corticoid because there was no significant structural disarrangement. Sun et $a .^{25}$ also showed protection of the cochlear morphology. In the CDDP + multidose dexamethasone group, a virtually normal stria vascularis was seen when compared with the control (serum) group; in addition, the quantification of residual hair cells in this group was significantly higher than in the CDDP alone group. Waissbluth et al. ${ }^{26}$ demonstrated preservation of stria vascularis morphology with the use of systemic corticosteroids but did not have the same finding for OHCs, which were partially destroyed.

In relation to the $\mathrm{OHCs}$, the result found was similar to that described by Waissbluth et $a l^{26}$, as there was a statistically significant difference between the groups C $\times$ DEXA15 + CDDP. This demonstrated a lack of histological protection of the OHCs.

This study verified that the auditory lesion triggered by CDDP comes from the cochlear structures because there was no significant increase in interval IV in the treated animals. A similar result was observed by Rebert et $a{ }^{28}{ }^{28}$, as well as by Freitas ${ }^{23}$. However, in hamsters, Church et al. ${ }^{29}$ reported prolongation of the I-IV interval with the $15 \mathrm{mg} / \mathrm{kg}$ dose of CDDP, suggesting retrocochlear damage by this drug.

\section{Conclusion}

The use of dexamethasone at a dose of $15 \mathrm{mg} / \mathrm{kg} /$ day partially protected against CDDPinduced ototoxicity in rats, as determined by BERA evaluation and morphological evaluation.

\section{References}

1. Kelland LR, Farrell N. Platinum-based drugs in cancer therapy. Totowa: Humana Press; 2000.

2. Brock PR, Knight KR, Freyer DR, Campbell KC, Steyger PS, Blakley BW, Rassekh SR, Chang KW, Fligor BJ, Rajput K, Sullivan M, Neuwelt EA. Platinum-induced ototoxicity in children: a consensus review on mechanisms, predisposition, and protection, including a new International society of pediatric oncology Boston ototoxicity scale. J Clin Oncol. 2012;30(19):2408-17. doi: 10.1200/ jco.2011.39.1110.

3. SchweitzerVG. Cisplatin-induced ototoxicity: the effect of pigmentation and inhibitory agents. Laryngoscope. 1993;103(4):1-52. doi: 10.1288/00005537-199304000-00001.

4. Parnes LS, Sun AH, Freeman DJ. Corticosteroid pharmacokinetics in the inner ear fluids: an animal study followed 
by clinical application. Laryngoscope. 1999;109(7):1-17. doi: 10.1097/00005537199907001-00001.

5. Hamid M, Trune D. Issues, indications, and controversies regarding intratympanic steroid perfusion. Curr Opin Otolaryngol Head Neck Surg. 2008;16(5):434-40. doi: 10.1097/MOO.0b013e32830ce796.

6. Murphy D, Daniel SJ. Intratympanic dexamethasone to prevent cisplatin ototoxicity: a guinea pig model. Otolaryngol Head Neck Surg. 2011;145(3):452-7. doi: 10.1177/0194599811406673.

7. Fram RJ. Cisplatin and platinum analogues: recent advances. Curr Opin Oncol. 1992;4(6):1073-9. doi: 10.1097/00001622199212000-00012.

8. Rybak LP, Ramkumar V. Ototoxicity. Kidney Int. 2007;72(8):931-5. doi: 10.1038/ sj.ki.5002434.

9. Feghali JG, Liu W, van de Water TR. L-nacetyl-cysteine protection against cisplatininduced auditory neuronal and hair cell toxicity. Laryngoscope. 2001;111(7):114755. doi: 10.1097/00005537-20010700000005.

10.Minami SB, Sha SH, Schacht J. Antioxidant protection in a new animal model of cisplatin-induced ototoxicity. Hear Res. 2004;198(1-2):137-43. doi: 10.1016/j. heares.2004.07.016.

11.Kalkanis JG, Whitworth C, Rybak LP. Vitamin $E$ reduces cisplatin ototoxicity. Laryngoscope. 2004;114(3):538-42. doi: 10.1097/00005537-200403000-00028.

12. Horie RT, Sakamoto T, Nakagawa T, Ishihara T, Higaki M, Ito J. Stealth-nanoparticle strategy for enhancing the efficacy of steroids in mice with noise-induced hearing loss. Nanomedicine. 2010;5(9):1331-40. doi: $10.2217 / \mathrm{nnm} .10 .88$.

13. Hill GW, Morest DK, Parham K. Cisplatininduced ototoxicity: effect of intratympanic dexamethasone injections. Otol Neurotol. 2008;29(7):1005-11. doi: 10.1097/ MAO.0b013e31818599d5.

14.Barnes PJ, Karin M. Nuclear factor-kappaB: a pivotal transcription factor in chronic inflammatory diseases. N Engl J Med. 1997;336(15):1066-71. doi: 10.1056/ nejm199704103361506.

15.Lewis TS, Shapiro PS, Ahn NG. Signal transduction through MAP kinase cascades.
Adv Cancer Res. 1998;74:49-139. doi: 10.1016/S0065-230X(08)60765-4.

16.Guha M, O'Connell MA, Pawlinski R, Hollis A, McGovern P, Yan SF, Stern D, Mackman N. Lipopolysaccharide activation of the MEKERK1/2 pathway in human monocytic cells mediates tissue factor and tumor necrosis factor alpha expression by inducing Elk1 phosphorylation and Egr-1 expression. Blood. 2001;98(5):1429-39. doi: 10.1182/ blood.V98.5.1429.

17.Ghosh S, May MJ, Kopp EB. NF-kappa B and Rel proteins: evolutionarily conserved mediators of immune responses. Annu Rev Immunol. 1998;16:225-60. doi: 10.1146/ annurev.immunol.16.1.225.

18.Auphan N, DiDonato JA, Rosette C, Helmberg A, Karin M. Immunosuppression by glucocorticoids: inhibition of NF-kappa $B$ activity through induction of I kappa B synthesis. Science. 1995;270(5234):286-90. doi: $10.1126 /$ science.270.5234.286.

19.So H, Kim H, Lee JH, Park C, Kim Y, Kim E, Kim JK, Yun KJ, Lee KM, Lee HY, Moon SK, Lim DJ, Park R. Cisplatin cytotoxicity of auditory cells requires secretions of proinflammatory cytokines via activation of ERK and NF-kappaB. J Assoc Res Otolaryngol. 2007;8(3):338-55. doi: 10.1007/s10162007-0084-9.

20.Sockalingam R, Freeman S, Cherny TL, Sohmer H. Effect of high-dose cisplatin on auditory brainstem responses and otoacoustic emissions in laboratory animals. Am J Otol. 2000;21(4):521-7. PMID: 10912698.

21. Kamimura T, Whitworth CA, Rybak LP. Effect of 4-methylthiobenzoic acid on cisplatininduced ototoxicity in the rat. Hear Res. 1999;131(1-2):117-27. doi: 10.1016/S03785955(99)00017-9.

22. Tanaka F, Whitworth CA, Rybak LP. Round window $\mathrm{pH}$ manipulation alters the ototoxicity of systemic cisplatin. Hear Res. 2004;187(1-2):44-50. doi: 10.1016/S03785955(03)00330-7.

23. Freitas MR. The role of apoptosis in cisplatin-induced ototoxicity in rats. Braz J. Otorhinolaryngol. 2009;75:745-52. doi: 10.1590/S1808-86942009000500022.

24.Lui HJ, Dong MM, Chi FL. Dexamethasone pharmacokinetics in guinea pig inner ear perilymph. ORL J Otorhinolaryngol 
Relat Spec. 2006;68(2):93-8. doi: 10.1159/000091210.

25.Sun C, Wang X, Chen D, Lin X, Yu D, Wu H. Dexamethasone loaded nanoparticles exert protective effects against Cisplatin-induced hearing loss by systemic administration. Neurosci Lett. 2016;619:142-8. doi: 10.1016/j.neulet.2016.03.012.

26.Waissbluth S, Salehi P, He X, Daniel SJ. Systemic dexamethasone for the prevention of cisplatin-induced ototoxicity. Eur Arch Otorhinolaryngol. 2013;270(5):1597-605. doi: 10.1007/s00405-012-2150-0.

27.Salehi P, Akinpelu OV, Waissbluth S, Peleva E, Meehan B, Rak J, Daniel SJ. Attenuation of cisplatin ototoxicity by otoprotective effects of nanoencapsulated curcumin and dexamethasone in a guinea pig model. Otol Neurotol. 2014;35(7):1131-9. doi: 10.1097/ mao.0000000000000403.

28. Rebert CS, Pryor GT, Frick MS. Effects of vincristine, maytansine, and cis-platinum on behavioral and electrophysiological indices of neurotoxicity in the rat. J Appl Toxicol. 1984;4(6):330-8. doi: 10.1002/ jat.2550040610.

29. Church MW, Kaltenbach JA, Blakley BW, Burgio DL. The comparative effects of sodium thiosulfate, diethyldithiocarbamate, fosfomycin and WR-2721 on ameliorating cisplatin-induced ototoxicity. Hear Res. 1995;86(1-2):195-203. doi: 10.1016/03785955(95)00066-D.

\section{Correspondence:}

Isabelle Oliveira Jatai Capelo

Rua Dr. Batista de Oliveira, 668/1903

60176-032 Fortaleza - CE Brasil

Tel.: (55 85)98752-5547

bellejatai@yahoo.com.br

Received: June 08, 2017

Review: Aug 10, 2017

Accepted: Sept 13, 2017
Conflict of interest: none

Financial source: none
${ }^{1}$ Research performed at Laboratory of Surgery Service, Universidade Federal do Ceará (UFC), Fortaleza-CE, Brazil. 